\title{
RETRACTED: Twenty-Three Species of Hypobarophilic Bacteria Recovered from Diverse Ecosystems Exhibit Growth under Simulated Martian Conditions at $0.7 \mathrm{kPa}$
}

\author{
Andrew C. Schuerger ${ }^{1}$ and Wayne L. Nicholson ${ }^{2}$
}

\begin{abstract}
On 27 April 2016, after acceptance and publication of the above article, the senior author learned that the University of Florida (UF) Institutional Review Board (IRB) review and approval process for using human subjects in a minimal risk scientific research project was not properly followed. Since that date, the senior author has worked closely with the UF/IRB team, the editorial staff at the journal Astrobiology, and the publisher Mary Ann Liebert, Inc. to discuss and rectify the situation. At the time the research was performed (Nov. 2012 through March 2013), the three human volunteers mentioned in the article were clearly informed of the intended use of the samples outlined in the study, were informed that the results (if meritorious) would be published in a peer-reviewed scientific journal, and were informed that all samples would be destroyed by autoclaving and then incineration at the conclusion of the research. No identifiable metadata related to the volunteers was recorded. The authors stand by the original results and interpretations of the research, but must now retract the paper because the guidelines for working with human subjects were not followed. We apologize for the error, and look forward to reworking the manuscript into a new paper with the human data removed, and submitting it to an appropriate peer-reviewed astrobiology publication venue.

Astrobiology commends Dr. Schuerger for his honesty and transparency.
\end{abstract}

\section{Introduction}

$\mathbf{E}$ ARTH's GLOBAL AVERAGE atmospheric pressure at sea level is $101.33 \mathrm{kPa}$ (i.e., $1013.3 \mathrm{mbar}$ ) and decreases with altitude to below $1 \mathrm{~Pa}$ above $80 \mathrm{~km}$. In contrast, high-pressure environments up to $1.1 \mathrm{GPa}$ (i.e., 1086 bars) are present in deep-sea sites like the bottom of the Mariana Trench in the Pacific Ocean. Numerous studies (see reviews in Michiels et al., 2008) have explored how microorganisms respond to increasing pressure, and such piezophiles have been reported capable of metabolic activity up to $16 \mathrm{GPa}$ (e.g., Shewanella oneidensis and Escherichia coli; Sharma et al., 2002). However, what about low pressure? Are microorganisms metabolically active and capable of growth at significantly reduced atmospheric pressures? The question is relevant for studies into metabolic activity and growth of microorganisms in our terrestrial atmosphere and for extraterrestrial environments with low-pressure atmospheres such as Mars.

From a terrestrial perspective, a few studies have begun to explore metabolic activity of bacteria under partially simulated cloud and tropospheric conditions (e.g., Amato et al., 2007; Santl-Temkiv et al., 2013; Vaitilingom et al., 2013). However, simulations of microbial activity under cloud or tropospheric conditions often have ignored low pressure as an experimental factor. Several exceptions can be noted. First, vegetative cells, but not endospores, of six Bacillus spp. ex- hibited growth down to $2.5 \mathrm{kPa}$ in an Earth-normal $\mathrm{O}_{2} / \mathrm{N}_{2}$ atmosphere (Schuerger and Nicholson, 2006). Recent papers have demonstrated microbial growth and up-regulation of the des-desKR system encoding membrane fatty acid desaturase (Fajardo-Cavazos et al., 2012), evolution (Nicholson et al., 2010), and altered global gene expression (Waters et al., 2014) of Bacillus subtilis strains cultivated under Earth-normal $\mathrm{O}_{2} /$ $\mathrm{N}_{2}$ atmospheres at $5 \mathrm{kPa}$. A pressure range of 2.5 to $5 \mathrm{kPa}$ is equivalent to altitudes of 20 to $25 \mathrm{~km}$, respectively, in Earth's atmosphere.

From an extraterrestrial perspective, two recent studies have demonstrated growth of a few bacterial species in low-pressure environments $<2.5 \mathrm{kPa}$, including seven permafrost isolates and nine type-strains of Carnobacterium spp. (Nicholson et al., 2013) and a single type-strain of Serratia liquefaciens (Schuerger et al., 2013) that exhibited vigorous to moderate growth, respectively, on semisolid media incubated under Mars surface conditions of $0.7 \mathrm{kPa}$, $0^{\circ} \mathrm{C}$, and $\mathrm{CO}_{2}$-enriched anoxic atmosphere. Taken together, a number of studies (Schuerger and Nicholson, 2006; Schuerger et al., 2006, 2013; Nicholson et al., 2010, 2013; Fajardo-Cavazos et al., 2012; Waters et al., 2014) have demonstrated that of over 150 bacterial strains tested under a range of decreasing pressures, most bacteria grew nominally between 10.0 and $101.3 \mathrm{kPa}$, with decreased growth rates noted for most bacteria below $10.0 \mathrm{kPa}$, and the cessation of

\footnotetext{
${ }^{1}$ Department of Plant Pathology and ${ }^{2}$ Department of Microbiology and Cell Science, University of Florida, Gainesville, Florida.
} 
growth for most species at $2.5-3.5 \mathrm{kPa}$. Only a few species including Carnobacterium spp. $(0.7 \mathrm{kPa}$; Nicholson et al., 2013), Pseudomonas fluorescens (1.5 kPa; Schuerger, unpublished data), and Serratia liquefaciens $(0.7 \mathrm{kPa}$; Schuerger et al., 2013) exhibited growth below $2.5 \mathrm{kPa}$.

The primary objective of the current research was to determine whether other archaea, bacteria, or fungi from soil and non-soil ecological niches were capable of growth at pressures that approach those found in the middle stratosphere on Earth and the surface of Mars (i.e., 0.7-1.0 kPa). In a recent review, Smith (2013) argued successfully that studies into microbial survival, growth, and evolution in the terrestrial middle to upper atmosphere are likely to inform and guide planetary protection and astrobiology studies into potential microbial activity on Mars. The work described here was designed to screen for microbial activity under conditions relevant to the surface of Mars but is applicable to microbial high-altitude studies in Earth's atmosphere.

We use the terms hypobarophile to denote microorganisms capable of metabolic activity and growth at pressures $\leq 1-2 \%$ sea level pressure $(\leq 1-2 \mathrm{kPa})$ and mesobarophile to denote microorganisms that can only grow at pressures between 2.5 and $101.3 \mathrm{kPa}$.

\section{Materials and Methods}

\subsection{Sample sources}

Soils were collected by several individuals from nine locations including Arctic, Antarctic, desert, alpine, and Kennedy Space Center sites (Table 1). Soils were shipped and stored at $-15^{\circ} \mathrm{C}$ (most soils) or $-70^{\circ} \mathrm{C}$ (permafrost soils only) until processed. All soils were collected from surface profiles to a depth of 1-2 cm, except the Siberian permafrost soils, which were collected from permafrost boreholes (Nicholson et al., 2013).

Non-soil samples were collected from diverse ecological settings. Several local volunteers provided saliva, handrinsate, forearm hair, or scalp hair for assays. In addition, we collected plant leaf imprints on selective media, seawater samples from the surf along the Florida coast at the Merritt Island National Refuge, and swabs of bench tops in one of our labs (ACS). Saliva samples were collected as $1 \mathrm{~mL}$ aliquots in $15 \mathrm{~mL}$ sterile polystyrene tubes. Saliva samples (100 $\mu \mathrm{L} /$ plate) were then plated directly onto $0.5 \times$ trypticase soy agar (TSA). Hand rinsates were collected by submerging hands in $500 \mathrm{~mL}$ of sterile deionized water for $60 \mathrm{~s}$ (with agitation), the water filtered through sterile $0.45 \mu \mathrm{m}$ nitrocellulose filters (Whatman, 7190-004, GE Healthcare Life Sciences, Kent, England), filters inverted on TSA, and incubated as described below. Hair was collected aseptically in the lab from volunteers and placed directly on selective media. Seawater was collected, and then three $500 \mathrm{~mL}$ aliquots were filtered through separate sterile $0.45 \mu \mathrm{m}$ nitrocellulose filters, the filters inverted on selective media, and incubated at $0.7 \mathrm{kPa}$, as described below. After 7 days, the filters from both hand rinsate and seawater samples were removed and discarded. The filter cultures were then returned to the low-pressure conditions. Leaf imprints were created by randomly selecting fully expanded true leaves from a coral bean plant (Erythrina herbacea L.) adjacent to

Table 1. Numbers of Hypobarophiles Recovered from Diverse Soil and Non-Soil Niches

\begin{tabular}{|c|c|c|c|c|c|c|}
\hline Samples & & nit & $\begin{array}{c}\text { ypobarophiles } \\
0.7 \mathrm{kPa}, \mathrm{O}^{\circ} \mathrm{C} \text {, } \\
\text { and } \mathrm{CO}_{2} \\
\left(1 / 2 T S A^{\mathrm{b}}\right)\end{array}$ & $\begin{array}{c}\text { No. culturable } \\
\text { bacteria at } \\
101.3 \mathrm{kPa} \text {, } \\
25^{\circ} \mathrm{C} \text {, and } \\
\mathrm{O}_{2}(1 / 2 \mathrm{TSA})\end{array}$ & $\begin{array}{c}\text { No. culturable } \\
\text { fungi at } \\
101.3 \mathrm{kPa}, \\
25^{\circ} \mathrm{C} \text {, and } \\
\mathrm{O}_{2}\left(P D A T C^{\mathrm{c}}\right)\end{array}$ & $\begin{array}{c}\text { Source of } \\
\text { soils or samples }\end{array}$ \\
\hline \multicolumn{7}{|l|}{ Soils } \\
\hline Atacama Desert, Chile & & $\mathrm{g}$ & 0 & $1.3 \times 10^{2}$ & $5.0 \times 10^{1}$ & C.P. McKay \\
\hline Battleship Promontory, Antarctica & 2 & $\begin{array}{l}0 \\
\mathrm{~g}\end{array}$ & 0 & $5.8 \times 10^{1}$ & 0 & C.P. McKay \\
\hline Mt. Baker, Washington & 2 & $\begin{array}{l}\text { g } \\
\text { g }\end{array}$ & $1.9 \times 10^{2}$ & $1.2 \times 10^{8}$ & $7.2 \times 10^{2}$ & D.J. Smith \\
\hline Colour Lake, Axel Heiberg Island & 2 & $\begin{array}{l}\text { g } \\
\text { g }\end{array}$ & $5.1 \times 10^{4}$ & $1.0 \times 10^{7}$ & $2.2 \times 10^{2}$ & C.P. McKay \\
\hline Devon Island, Canada & 2 & $\begin{array}{l}\text { g } \\
\mathrm{g}\end{array}$ & $2.5 \times 10^{2}$ & $3.4 \times 10^{4}$ & 0 & R.J. Ferl \\
\hline Mojave Desert, Californi & 1 & $\begin{array}{l}\text { g } \\
\text { g }\end{array}$ & 0 & $6.8 \times 10^{5}$ & $2.0 \times 10^{3}$ & C.P. McKay \\
\hline PHSF, KSC, FL & 4 & $\begin{array}{l}0 \\
\mathrm{~g}\end{array}$ & 0 & $9.8 \times 10^{6}$ & $3.7 \times 10^{5}$ & A.C. Schuerger \\
\hline Río Tinto, Spain & 1 & $\begin{array}{l}0 \\
\mathrm{~g}\end{array}$ & 0 & $3.5 \times 10^{3}$ & $3.4 \times 10^{3}$ & V. Parro \\
\hline Siberian permafrost, Russia & 6 & $\begin{array}{l}g \\
\mathrm{~g}\end{array}$ & $2.8 \times 10^{4}$ & $1.5 \times 10^{8}$ & 0 & K. Krivushin \\
\hline \multicolumn{7}{|l|}{ Non-soils } \\
\hline Coral bean leaves, KSC, FL & 2 & $10 \mathrm{~cm}^{2}$ & 0 & $5.0 \times 10^{1}$ & $2.5 \times 10^{1}$ & A.C. Schuerger \\
\hline Forearm hair, human & 1 & & 0 & $\mathrm{NT}^{\mathrm{e}}$ & NT & 2 volunteers \\
\hline Lab benches, SLSL ${ }^{\mathrm{f}}, \mathrm{KSC}, \mathrm{FL}$ & 3 & $100 \mathrm{~cm}^{2}$ & 0 & $4.3 \times 10^{1}$ & 0 & A.C. Schuerger \\
\hline Saliva, human & 2 & $\mathrm{~mL}$ & $5.0 \times 10^{2}$ & $6.4 \times 10^{6}$ & 0 & 3 volunteers \\
\hline Seawater, KSC, FL & 1 & $250 \mathrm{~mL}$ & 2 & NT & NT & A.C. Schuerger \\
\hline Scalp hair, human & 3 & & 0 & NT & NT & 2 volunteers \\
\hline Washed hands, human & 1 & $250 \mathrm{~mL}$ & 2 & NT & NT & 3 volunteers \\
\hline
\end{tabular}

${ }^{\mathrm{a}}$ Units used in the population numbers for hypobarophiles, bacteria, and fungi recovered from various sources. $\mathrm{g}=\mathrm{grams}$; $\mathrm{cm}=$ centimeters; $\mathrm{mL}=$ milliliters.

${ }^{\mathrm{b}} \mathrm{TSA}=$ trypticase soy agar.

${ }^{c}$ PDATC = potato dextrose agar supplemented with Tergitol NP-10 at $1 \mathrm{~mL} / \mathrm{L}$ and chlortetracycline $\mathrm{HCl}$ at $50 \mathrm{mg} / \mathrm{L}$.

${ }^{\mathrm{d}} \mathrm{PHSF}=$ Payload Hazardous Servicing Facility, Kennedy Space Center, FL.

${ }^{\mathrm{e}} \mathrm{NT}=$ not tested.

${ }^{\mathrm{f}} \mathrm{SLSL}=$ Space Life Sciences Lab, Kennedy Space Center, FL. 
the Space Life Sciences Lab (SLSL; Merritt Island, FL). The leaves were inverted and the adaxial surfaces pressed into agar surfaces for $30 \mathrm{~s}$. The leaf imprints were then incubated at $0.7 \mathrm{kPa}$, as described below.

\subsection{Soil dilution plate assays}

All soils were removed from cold storage and allowed to partially thaw for sample removal. One gram of each soil was added to separate $125 \mathrm{~mL}$ flasks containing $25 \mathrm{~mL}$ of autoclaved $0.1 \%$ water agar, and vigorously mixed with magnetic stir bars for $10 \mathrm{~min}$. The $0.1 \%$ water-agar mixtures were semiliquid when stirred and acted to keep soil particles in suspension. The soil/agar suspensions (200 $\mu \mathrm{L} /$ plate) were plated directly onto double-thick $(30 \mathrm{~mL}$ per plate) 0.5× TSA (BD Difco, Becton, Dickinson, and Company, Sparks, MD, USA) or R2A agar (BD Difco) plates.

\subsection{Hypobaric conditions}

After plating, all samples were immediately placed under low-pressure $(0.7 \mathrm{kPa})$, low-temperature $\left(0^{\circ} \mathrm{C}\right)$, and $\mathrm{CO}_{2^{-}}$ enriched anoxic (low $\mathrm{ppO}_{2}$ ) conditions (henceforth called low-PTA conditions), as described previously (Schuerger et al., 2013). The hypobaric assays were conducted in 4-L polycarbonate desiccators (model 08-642-7, Fisher Scientific, Pittsburg, PA, USA) fitted with $0.45 \mu$ m HEPA filters (Whatman, model 6723-5000) on the top and bottom sections for sterile operation of the vacuum and repressurization processes (Schuerger et al., 2013). The desiccators were connected to an external pump and controller (model PU842 , KNF Neuberger, Trenton, NJ, USA) capable of holding pressure at $0.7 \mathrm{kPa}( \pm 0.1 \mathrm{kPa})$.

Anoxic conditions within the desiccators were achieved by first placing four anaerobic sachets (Remel AnerobicPack, Fisher Scientific) into the vacuum chambers, closing the desiccators, and flushing them with researchgrade $\mathrm{CO}_{2}$ for $5 \mathrm{~min}$. The samples were then held in the lowPTA conditions for 28 days, with the anaerobic sachets changed every 7 days. After 4 weeks, the plates were inspected visually for observable colonies (Round 1 hypobaric assay). Visible colonies were picked, streaked-purified on the same media ( $0.5 \times$ TSA or R2A) used in the Round 1 assays, and incubated at lab conditions of $101.3 \mathrm{kPa}, 24^{\circ} \mathrm{C}$, and Earth-normal $\mathrm{O}_{2} / \mathrm{N}_{2}$ atmosphere. The pure cultures were then applied to fresh media with heat-sterilized loops and incubated under low-PTA conditions for 14 days (Round 2). Single isolated colonies from Round 2 were picked, restreaked onto fresh plates of the same medium, and incubated a third time under low-PTA conditions for 14 days (Round 3). Isolates that grew through Rounds 1-3 were given strain designations (Table 2), stored at $-70^{\circ} \mathrm{C}$ as frozen glycerol stocks, and characterized further.

The Siberian permafrost isolates were then processed for a separate study on the effects of low temperature on colony morphology and pigmentation. Permafrost hypobarophiles were grown on $0.5 \times \mathrm{TSA}$ for 7 days at $15^{\circ} \mathrm{C}$ under either an anoxic atmosphere $\left(\mathrm{CO}_{2}\right.$-enriched anaerobic chambers) or a lab-normal oxygenated atmosphere $\left(\mathrm{O}_{2} / \mathrm{N}_{2} ; 21 \% / 78 \%\right.$, respectively). Bacterial colonies were imaged at identical magnifications and lighting conditions.

If either $0.5 \times \mathrm{TSA}$ or R2A media were placed in the desiccators and pumped directly from 101.3 to $0.7 \mathrm{kPa}$, the media would often bubble, crack, and deform. Thus, to permit the outgassing of dissolved lab air from the agar, a three step pump-down procedure was developed. Media in Petri dishes were placed in the 4-L desiccators as described above, set in the $0^{\circ} \mathrm{C}$ incubator, pumped to a range of $7.5-10.0 \mathrm{kPa}$, and allowed to equilibrate for $30 \mathrm{~min}$. Then the vacuum chamber was pumped to $2.5 \mathrm{kPa}$ for an additional $30 \mathrm{~min}$. After a total elapsed time of $1 \mathrm{~h}$ for outgassing, the vacuum chambers were then allowed to equilibrate at $0.7 \mathrm{kPa}$.

\section{4. $16 S$ rRNA sequencing}

Bacteria that demonstrated growth at $0.7 \mathrm{kPa}$ through Rounds 1-3 were labeled as hypobarophiles, and the $16 \mathrm{~S}$ rRNA genes were sequenced from purified strains. DNA was extracted from each strain with the UltraClean Microbial DNA Isolation Kit (12224-50, MoBio Laboratories, Inc., Carlsbad, CA, USA) and PCR amplified according to the protocols of Benardini et al. (2003). All PCR amplicons were then cleaned with the QIAquick PCR Purification Kit (model 28104, QIAGEN Sciences, Valencia, CA, USA). Universal bacterial primers for the 16S rRNA region were B27F (5'-GAGTTTGATCMTGGCTCAG-3') and B1512R (5'-AAGGAGGTGATCCANCCRCA-3') (Lueders et al., 2004). Purified PCR amplicons were Sanger sequenced at the Interdisciplinary Center for Biotechnology Research (ICBR) at the University of Florida (Gainesville, FL, USA).

Strains were assigned taxonomic affiliations based on $\geq 97.5 \%$ similarities (Stackebrandt et al., 2002) to published entries in the Ribosomal Database Project (RDP) library (Release 10, Update 31) (Cole et al. 2009) [http://rdp.cme msu.edu]. Nucleotide sequences have been deposited in GenBank [http://ncbi.nlm.nih.gov/genbank; National Center for Biotechnology Information, USA] under the accession numbers given in Table 2.

\subsection{Growth of type-strains for the genus Serratia under low-PTA conditions}

Schuerger et al. (2013) demonstrated that the type-strain Serratia liquefaciens (ATCC 25792) was capable of growth under low-PTA conditions at $0.7 \mathrm{kPa}$, and Nicholson et al. (2013) demonstrated the same capability for nine type-strains in the genus Carnobacterium. To determine whether a genuswide capability for low-pressure growth similar to Carnobacterium existed for Serratia, eight type-strains in the genus Serratia were incubated under low-PTA conditions.

Cultures of Serratia type-strains (Table 3) were grown under low-PTA conditions on $0.5 \times$ TSA for 35 days. Four additional bacterial strains were grown at $0.7 \mathrm{kPa}$ as either positive controls (Carnobacterium inhibens subsp. gilichinskyi, strain WN1359; Nicholson et al., 2015) or as negative controls (Bacillus subtilis, Escherichia coli, and Sporosarcina aquamarina; Schuerger et al., 2013). Growth for each strain was recorded using a simple rating system that ranked growth relative to the size and morphology of the control strains at Earth-normal lab conditions of $101.3 \mathrm{kPa}, 30^{\circ} \mathrm{C}$, and Earthnormal $\mathrm{O}_{2} / \mathrm{N}_{2}$ atmosphere (21\%/78\%, respectively). Ratings in Table 3 are given as [+] indicating weak growth to [++1+] indicating vigorous growth (see Fig. 1 ); $[-]=$ no growth. All bacteria with a recorded [-] growth response after 35 days were returned to lab conditions for $48 \mathrm{~h}$. In all cases, the strains 
Table 2. Hypobarophile Bacteria Recovered from SoIl and Non-Soil Niches

\begin{tabular}{|c|c|c|c|c|c|c|}
\hline Source $e^{\mathrm{a}, \mathrm{b}, \mathrm{c}}$ & $\begin{array}{l}\text { 16S rRNA RDP } \\
\text { identification }\end{array}$ & Strain \# & $\begin{array}{c}\text { GenBank } \\
\text { accession \# }\end{array}$ & $\begin{array}{c}\text { RDP } \\
\text { closest } \\
\text { match }\end{array}$ & Phylum & Family \\
\hline \multicolumn{7}{|l|}{ Soil hypobarophiles } \\
\hline Colour Lake soil & Bacillus sp. & ASB-86 & KR857399 & 0.844 & Firmicutes & Bacillaceae \\
\hline Colour Lake soil & Bacillus sp. & ASB-88 & KR857401 & 0.880 & Firmicutes & Bacillaceae \\
\hline Colour Lake soil & Clostridium sp. & ASB-85 & KR857398 & 0.953 & Firmicutes & Clostridiaceae \\
\hline Colour Lake soil & Clostridium sp. & ASB-95 & KR857406 & 0.921 & Firmicutes & Clostridiaceae \\
\hline Colour Lake soil & Cryobacterium sp. & ASB-87 & KR857400 & 0.952 & Firmicutes & Microbacteriaceae \\
\hline Colour Lake soil & Cryobacterium sp. & ASB-84 & KR857397 & 0.954 & Firmicutes & Microbacteriaceae \\
\hline Colour Lake soil & Paenibacillus antarc & ASB-59 & KR857372 & 0.987 & Firmicutes & Paenibacillaceae \\
\hline Colour Lake soil & Paenibacillus antarcticus & ASB-67 & KR857380 & 0.989 & Firmicutes & Paenibacillaceae \\
\hline Colour Lake soil & Paenibacillus antarcticus & ASB-90 & KR857403 & 0.985 & Firmicutes & Paenibacillaceae \\
\hline Colour Lake soil & Paenibacillus antarcticus & ASB-94 & KR857405 & 0.992 & Firmicutes & Paenibacillaceae \\
\hline Colour Lake soil & Paenibacillus antarcticus & ASB & KR857409 & 0.990 & Firmicutes & Paenibacillaceae \\
\hline Colour Lake soil & Paenibacillus antarcticus & ASB-99 & KR857410 & 0.995 & Firmicutes & Paenibacillaceae \\
\hline Colour Lake soil & $\begin{array}{l}\text { Paenibacillus } \\
\quad \text { macquariensis }\end{array}$ & ASB-55 & 7368 & 0.986 & Firmicutes & Paenibacillaceae \\
\hline Colour Lake soil & Paenibacillus sp. & ASB-56 & 7369 & 1.000 & & Paenib \\
\hline Colo & Paenibacillus sp. & A & 70 & 0.929 & $\mathrm{~F}$ & Paen \\
\hline Colour Lake soil & Paenibacillus sp. & ASB-66 & KR857379 & 0.935 & Firm & Paenibacillaceae \\
\hline Colour Lake soil & Paenibacillus sp. & ASB-68 & KR857381 & 0.996 & Firmicutes & Paenibacillaceae \\
\hline Colour Lake soil & Paenibacillus sp. & ASB-91 & KR857404 & 0.965 & Firmicutes & Paenibacillaceae \\
\hline Colour Lake soil & Rhodococcus qingshe & ASB-89 & KR857402 & 1.000 & Actinobacteria & Norcardiaceae \\
\hline Colour Lake soil & Streptomyces aureus & ASB-61 & KR857374 & 0.980 & Actinobacteria & Streptomycetaceae \\
\hline Colour Lake soil & Streptomyces aureus & ASB-62 & KR857375 & 0.980 & Actinobacteria & Streptomycetaceae \\
\hline Colour Lake soil & Streptomyces aureus & ASB-63 & KR857376 & 0.978 & Actinobacteria & Streptomycetaceae \\
\hline Colour Lake soil & Streptomyces aureus & ASB-64 & 7377 & 0.976 & Actinobacteria & Streptomycetaceae \\
\hline Colour La & Streptomyces vinaceus & AS & KR857378 & 0.995 & Actinobacteria & ycetaceae \\
\hline Devon Island soil & Paenibacillus sp. & AS & 7371 & 0.988 & Firmicutes & bacillaceae \\
\hline Devo & Paenibacillus sp. & AS & 382 & 0.996 & Fir & $\mathrm{Pa}$ \\
\hline Devo & Paenibacillus sp. & AS & 83 & 0.991 & Fir & $\mathrm{Pa} \in$ \\
\hline Dev & Paenibacillus sp. & & 84 & 0.988 & & $\mathrm{~Pa}$ \\
\hline an permafrost, \#42 & Carnobacterium $\mathrm{s}$ & & KR857411 & & & Carnobacteriaceae \\
\hline Siberian permafrost, \#42 & Carnobacteriun & WN-1501 & KR857427 & 0.996 & & Carnobacteriaceae \\
\hline Siberian permafrost, \#8 & Carnobacterium sp. & WN-1484 & KR857412 & 0.984 & & Carnobacteriaceae \\
\hline Siberian permafrost, \#8 & Carnobacterium & WN-1490 & KR857418 & 0.998 & Firmicutes & Carnobacteriaceae \\
\hline Siberian permafrost, \#9 & Carnobacterium & WN-1491 & KR857419 & 0.986 & Firm & Carnobacteriaceae \\
\hline Siberian permafrost, \#9 & Cryobacteriur & $3-53$ & KR857366 & 0.965 & Firm & Microbacteriaceae \\
\hline Siberian permafrost, \#9 & Cry & ASB-60 & 7373 & 0.995 & cutes & Microbacteriaceae \\
\hline Siberian permafrost, \#35 & Cryobacterium sp. & WN-1504 & KR857430 & 0.996 & Firmicutes & Microbacteriaceae \\
\hline Siberian permafrost, \#8 & Cryobacterium sp. & WN-1485 & 7413 & 0.972 & Firmicutes & Microbacteriaceae \\
\hline Siberian permafrost, \#9 & Cryobacterium sp. & WN-1502 & KR857428 & 0.980 & Firmicutes & Microbacteriaceae \\
\hline $\begin{array}{l}\text { Siberian permafrost soil, } \\
\quad \# 42\end{array}$ & $\begin{array}{l}\text { Exiguobacterium } \\
\text { sibiricum }\end{array}$ & ASB-51 & KR857364 & 1.000 & Firmicutes & $\begin{array}{l}\text { Bacillales_Incertae } \\
\text { Sedis XII }\end{array}$ \\
\hline Siberian permafrost, & cterium & WN-1486 & KR857414 & 0.991 & cutes & $\begin{array}{l}\text { Bacillales_Incertae } \\
\text { Sedis XII }\end{array}$ \\
\hline & $E x$ & WN-1488 & KR857416 & 0.991 & Firmicutes & $\begin{array}{l}\text { Bacillales_Incertae } \\
\text { Sedis XII }\end{array}$ \\
\hline Siberian permafrost, & $\begin{array}{l}\text { Exiguc } \\
\text { sibi }\end{array}$ & WN-1492 & KR857420 & 1.000 & Firmicutes & $\begin{array}{l}\text { Bacillales_Incertae } \\
\text { Sedis XII }\end{array}$ \\
\hline Siberian permafrost, \#9 & $\begin{array}{l}\text { Exiguobacterium } \\
\text { sibiricum }\end{array}$ & WN-1493 & KR857421 & 1.000 & Firmicutes & $\begin{array}{l}\text { Bacillales_Incertae } \\
\text { Sedis XII }\end{array}$ \\
\hline Sil & Trichococcus past & & & & & Carnobacteriaceae \\
\hline Siberian permafrost, \#35 & Trichococcus collinsii & WN-1503 & KR857429 & 0.996 & Firmicutes & Carnobacteriaceae \\
\hline Siberian permafrost, \#35 & Trichococcus collinsii & WN-1505 & KR857431 & 0.998 & Firmicutes & Carnobacteriaceae \\
\hline Siberian permafrost, \#35 & Trichococcus pasteurii & WN-1494 & KR857422 & 0.986 & Firmicutes & Carnobacteriaceae \\
\hline Siberian permafrost, \#35 & Trichococcus pasteurii & WN-1497 & KR857423 & 0.992 & Firmicutes & Carnobacteriaceae \\
\hline Siberian permafrost, \#35 & Trichococcus pasteurii & WN-1498 & KR857424 & 0.984 & Firmicutes & Carnobacteriaceae \\
\hline Siberian permafrost, \#35 & Trichococcus pasteurii & WN-1500 & KR857426 & 0.990 & Firmicutes & Carnobacteriaceae \\
\hline Siberian permafrost, \#35 & Trichococcus pasteurii & WN-1506 & KR857432 & 0.990 & Firmicutes & Carnobacteriaceae \\
\hline Siberian permafrost, \#45 & Trichococcus pasteurii & WN-1507 & KR857433 & 0.990 & Firmicutes & Carnobacteriaceae \\
\hline$\# 45$ & Trichococcus pasteurii & WN-1509 & 7435 & 0.991 & cutes & Carnobacteriaceae \\
\hline in permafrost, \#47 & Trichococcus pasteurii & WN-1508 & KR857434 & 0.989 & cutes & Carnobacteriaceae \\
\hline permafrost, \#8 & Trichococcus pasteurii & WN-1487 & & 0.993 & & Carnobacteriaceae \\
\hline Siberian permafrost, \#35 & Trichococcus sp. & WN-1499 & KR857425 & 0.985 & Firmicutes & Carnobacteriaceae \\
\hline
\end{tabular}


TABle 2. (CONTINUED)

\begin{tabular}{|c|c|c|c|c|c|c|}
\hline Source $e^{\mathrm{a}, \mathrm{b}, \mathrm{c}}$ & $\begin{array}{l}\text { 16S rRNA RDP } \\
\text { identification }\end{array}$ & Strain \# & $\begin{array}{c}\text { GenBank } \\
\text { accession \# }\end{array}$ & $\begin{array}{c}\text { RDP } \\
\text { closest } \\
\text { match }\end{array}$ & Phylum & Family \\
\hline \multicolumn{7}{|l|}{ Non-soil hypobarophiles } \\
\hline Hand rinsate, volunteer \#1 & Leuconostoc gelidum & ASB-96 & KR857407 & 0.992 & Firmicutes & Leuconostocaceae \\
\hline Hand rinsate, volunteer \#1 & Leuconostoc gelidum & ASB-97 & KR857408 & 0.992 & Firmicutes & Leuconostocaceae \\
\hline Saliva, volunteer \#1 & $\begin{array}{l}\text { Leuconostoc } \\
\text { gasicomitatum }\end{array}$ & ASB-72 & KR857385 & 1.000 & Firmicutes & Leuconostocaceae \\
\hline Saliva, volunteer \#1 & $\begin{array}{l}\text { Leuconostoc } \\
\text { gasicomitatum }\end{array}$ & ASB-74 & KR857387 & 1.000 & Firmicutes & Leuconostocaceae \\
\hline Saliva, volunteer \#1 & $\begin{array}{l}\text { Leuconostoc } \\
\text { gasicomitatum }\end{array}$ & ASB-75 & KR857388 & 0.991 & Firmicutes & Leuconostocaceae \\
\hline Saliva, volunteer \#1 & Leuconostoc gelidum & ASB-77 & KR857390 & 0.989 & Firmicutes & Leuconostocaceae \\
\hline Saliva, volunteer \#1 & Leuconostoc gelidum & ASB-78 & KR857391 & 1.000 & Firmicutes & Leuconostocaceae \\
\hline Saliva, volunteer \#1 & Leuconostoc gelidum & ASB-79 & KR857392 & 0.996 & Firmicutes & Leuconostocaceae \\
\hline Saliva, volunteer \#1 & Leuconostoc gelidum & ASB-80 & KR857393 & 0.996 & Firmicutes & Leuconostocaceae \\
\hline Saliva, volunteer \#1 & Leuconostoc gelidum & ASB-82 & KR857395 & 1.000 & Firmicutes & Leuconostocaceae \\
\hline Saliva, volunteer \#1 & Leuconostoc inhae & ASB-73 & KR857386 & 0.995 & Firmicutes & Leuconostocaceae \\
\hline Saliva, volunteer \#1 & Leuconostoc inhae & ASB-76 & KR857389 & 0.992 & Firmicutes & Leuconostocaceae \\
\hline Saliva, volunteer \#1 & Leuconostoc inhae & ASB-81 & KR857394 & 0.991 & Firmicutes & Leuconostocaceae \\
\hline Saliva, volunteer \#1 & Leuconostoc inhae & ASB-83 & KR857396 & 0.995 & Firmicutes & Leuconostocaceae \\
\hline \multicolumn{7}{|c|}{ Hypobarophiles in the genus Serratia } \\
\hline DSM & Serratia ficaria & 4569 & AJ233428 & & Proteobacteria & Enterobacteriaceae \\
\hline DSM & Serratia fonticola & 4576 & AJ233429 & n & Proteobacteria & Enterobacteriaceae \\
\hline ATCC & Serratia grimesii & 14460 & AX109622 & na & Proteobacteria & Enterobacteriaceae \\
\hline ATCC & Serratia liquefaciens & 27592 & AX109623 & na & Proteobacteria & Enterobacteriaceae \\
\hline DSM & Serratia plymuthica & 4540 & AJ233433 & na & Proteobacteria & Enterobacteriaceae \\
\hline ATCC & Serratia rubidaea & 27593 & AX109627 & na & Proteobacteria & Enterobacteriaceae \\
\hline
\end{tabular}

${ }^{a}$ Abbreviations: DSM = Leibniz Institute DSMZ-German Collection of Microorganisms and Cell Culture, Braunschweig, Germany; na $=$ not applicable; ATCC $=$ American Type Culture Collection, Manassas, VA, USA.

${ }^{\mathrm{b}}$ Sources for all samples are given in Table 1.

${ }^{\mathrm{c}}$ Siberian permafrost sample locations are given in the work by Nicholson et al. (2013).

Table 3. Growth of Species Type-Strains under Diverse Pressure, Temperature, and Gas Conditions

\begin{tabular}{|c|c|c|c|c|c|}
\hline Bacteria $^{\mathrm{a}}$ & & $\begin{array}{c}101.3 \mathrm{kPa} \\
30^{\circ} \mathrm{C} \\
\mathrm{O}_{2} / \mathrm{N}_{2}^{\mathrm{c}, \mathrm{d}}\end{array}$ & $\begin{array}{c}101.3 \mathrm{kPa} \\
\mathrm{O}^{\circ} \mathrm{C} \\
\mathrm{O}_{2} / \mathrm{N}_{2}\end{array}$ & $\begin{array}{c}101.3 \mathrm{kPa} \\
\mathrm{O}^{\circ} \mathrm{C} \\
\mathrm{CO}_{2}\end{array}$ & $\begin{array}{c}0.7 \mathrm{kPa} \\
\mathrm{O}^{\circ} \mathrm{C} \\
\mathrm{CO}_{2}\end{array}$ \\
\hline (1) Serratia ficaria & DSM 4569 & $++++^{\mathrm{e}}$ & +++ & $-f$ & ++ \\
\hline (2) S. fonticola & DSM 4576 & ++++ & ++++ & ++ & +++ \\
\hline (3) $S$. grimesii & ATCC 14460 & +++ & ++ & + & ++ \\
\hline (4) S. liquefaciens & ATCC 27592 & ++++ & ++++ & ++ & +++ \\
\hline (5) S. marcescens & ATCC 13880 & +++ & - & - & - \\
\hline (6) S. plymuthica & DSM 4540 & +++ & +++ & + & + \\
\hline (7) S. quinivorans & DSM 4597 & ++++ & +++ & + & ++ \\
\hline (8) S. rubidaea & ATCC 27593 & +++ & - & - & - \\
\hline (9) Bacillus subtilis & 168 & ++++ & - & - & - \\
\hline $\begin{array}{l}\text { (10) Carnobacterium inhibens } \\
\text { subsp. gilichinskyi }\end{array}$ & WN1359 & ++ & ++ & +++ & +++ \\
\hline (11) Escherichia coli & ATCC 35218 & ++++ & - & - & - \\
\hline (12) Sporosarcina aquamarina & SAFN-008 & ++++ & ++++ & - & - \\
\hline
\end{tabular}

${ }^{a}$ Numbers are used in Fig. 2 to delineate species grown under diverse pressure, temperature, and gas conditions.

${ }^{\mathrm{b}}$ Bacterial strains were obtained from (i) American Type Culture Collection (ATCC), (ii) Leibniz Institute DSMZ-German Collection of Microorganisms and Cell Cultures (DSM), (iii) W.L. Nicholson (168 and WN1359), and (iv) K. Venkateswaran (SAFN-008).

${ }^{c}$ Bacteria grown at $101.3 \mathrm{kPa}, 30^{\circ} \mathrm{C}$, and Earth-normal $\mathrm{O}_{2} / \mathrm{N}_{2}$ atmosphere were considered lab controls.

${ }^{\mathrm{d}}$ Assays for 0.7 and $101.3 \mathrm{kPa}$ were conducted for 35 days at the conditions indicated above.

${ }^{\mathrm{e}}$ Growth for each strain is recorded by plus signs indicating relative growth compared to the size of the control strain of Serratia liquefaciens ATCC 27592 at Earth-normal conditions. Ratings are from [+] indicating weak growth to [++++] indicating vigorous growth (see Fig. 2); [-]= no growth.

${ }^{\mathrm{f}}$ All bacteria with a recorded [-] growth response after 35 days grew normally (i.e., similar to lab controls) after cultures were transferred and incubated $48 \mathrm{~h}$ at $101.3 \mathrm{kPa}, 30^{\circ} \mathrm{C}$, and lab-normal $\mathrm{O}_{2} / \mathrm{N}_{2}$ atmosphere. In all cases, the bacteria grew normally under lab conditions. 
exhibiting negative growth at $0.7 \mathrm{kPa}$ responded to the lab conditions and grew normally (data not shown).

\section{Results}

\subsection{Isolation of hypobarophilic bacteria from soils}

Most soil samples grown under lab conditions of $101.3 \mathrm{kPa}, 20^{\circ} \mathrm{C}$, and Earth-normal $\mathrm{O}_{2} / \mathrm{N}_{2}$ conditions exhibited high diversities of bacteria and fungi on the agar surfaces of the soil-dilution plates. Figure 1A is an example of the high microbial diversity observed in four soils from Río Tinto, Spain; Atacama Desert, Chile; Colour Lake, Axel Heiberg Island, Canada; and Mt. Baker, Washington, USA. Both bacterial and fungal colonies were observed with multiple colony morphologies and pigmentations. In contrast, when hypobarophilic bacteria were recovered from soildilution plates maintained at $0.7 \mathrm{kPa}$, only white, translucent, and smooth-margined colonies were observed (Fig. 1B).

Total culturable bacteria in soils were estimated to range between $5.8 \times 10^{1}$ colony-forming units $(\mathrm{cfu}) / \mathrm{g}$ for the Antarctica soils from Battleship Promontory and $1.5 \times 10^{8} \mathrm{cfu} / \mathrm{g}$ of soil for the permafrost soils (Table 1). The total culturable fungi were estimated to range between zero for three coldtemperature soils from the Arctic or Antarctica and $3.7 \times 10^{5}$ $\mathrm{cfu} / \mathrm{g}$ for the Florida soils collected around the Payload Hazardous Servicing Facility at the Kennedy Space Center.

Hypobarophilic bacteria were recovered from soils in a range between zero cfu/g for one-half of the soils tested and $5.1 \times 10^{4} \mathrm{cfu} / \mathrm{g}$ of soils from Colour Lake (Table 1). Although fungal colonies were abundant in most soils grown under lab conditions (Fig. 1A), no fungi were observed to grow on either $0.5 \times$ TSA or R2A when plates were incubated at low-PTA conditions. In addition, all bacteria-like isolates recovered from TSA or R2A agar surfaces incubated under low-PTA conditions (Fig. 1B) were initially sequenced with $16 \mathrm{~S}$ primers. In all cases, $16 \mathrm{~S}$ amplicons were recovered and identified to species in the domain Bacteria. Thus, no archaea were recovered from TSA or R2A cultures incubated at low-PTA conditions.

Two hypobarophilic bacteria, Streptomyces aureus and $S$. vinaceus (Table 2), were isolated on three separate iterations of Round 1 assays with the Colour Lake soils but failed to grow under low-PTA conditions when streak-purified and tested in Round 2 assays. Although it is unknown whether the failure to grow in Round 2 assays for $S$. aureus and $S$. vinaceus was due to missing geochemical or biological components in the soil matrix, the Streptomyces species were consistently isolated in three separate Round 1 assays in the presence of the soil particles and undescribed biological communities. Thus, we list them here as presumptive hypobarophiles. All other bacteria that maintained growth at low-PTA conditions for Rounds 2 and 3 are listed as confirmed hypobarophiles (Table 2).

The focus for the current study was to recover and identify only culturable hypobarophilic species; thus no further work was attempted to isolate or identify culturable or nonculturable bacteria, archaea, and fungi that may have been concomitantly present in the soils. Furthermore, all hypobarophiles that grew under anoxic conditions at $0.7 \mathrm{kPa}$ were also able to grow under Earth-normal $\mathrm{ppO}_{2}$ at $101.3 \mathrm{kPa}$, except two strains of the obligate anaerobe Clostridium sp. (Table 2).
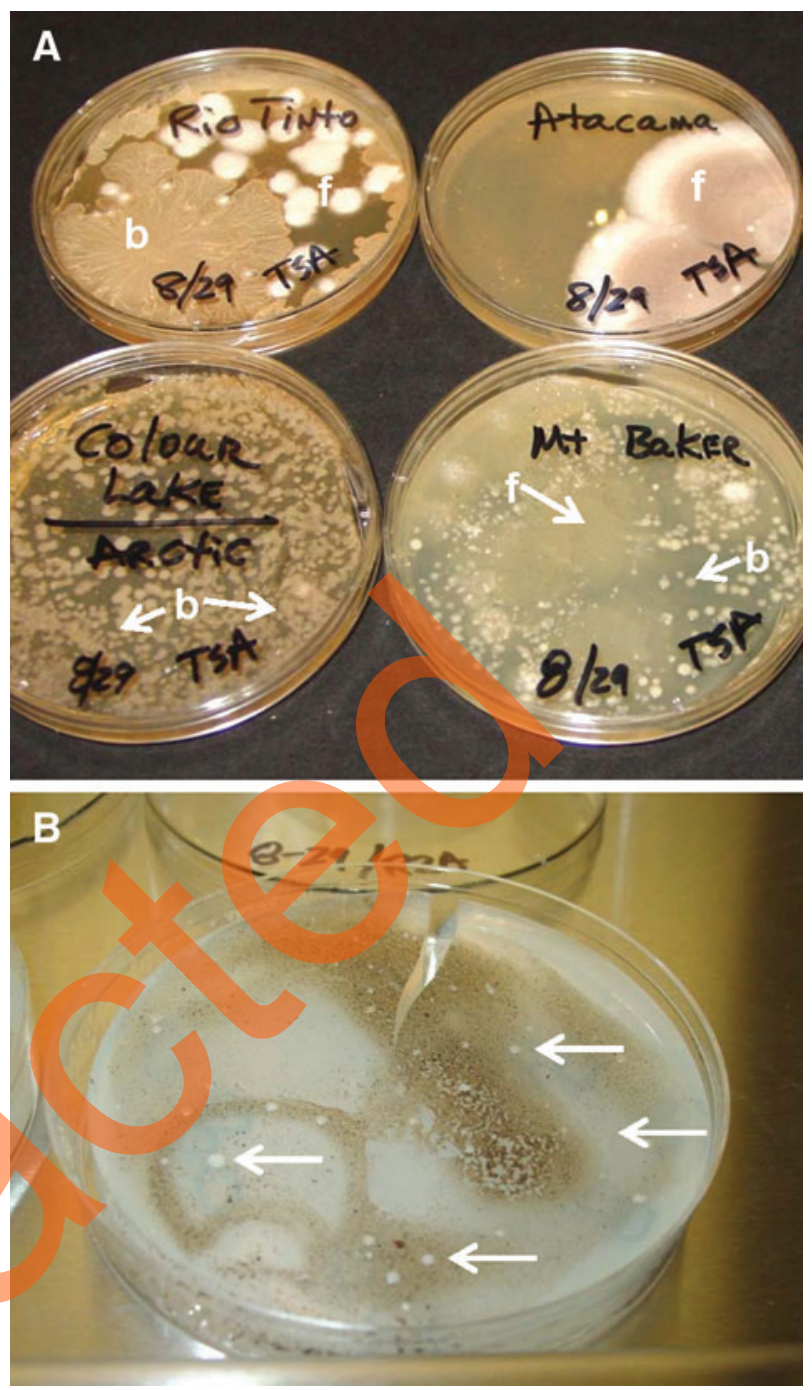

FIG. 1. Cultures of soil dilution plates incubated under lab (A) or martian (B) conditions. Soil dilution plates of soils were incubated on $0.5 \times$ TSA or R2A media under lab $\left(101.3 \mathrm{kPa}, 25^{\circ} \mathrm{C}\right.$, and $\left.21 \% \mathrm{ppO}_{2}\right)$ or martian $\left(0.7 \mathrm{kPa}, 0^{\circ} \mathrm{C}\right.$, and $\mathrm{CO}_{2}$-enriched anoxic atmosphere) conditions. (A) Bacterial (b) and fungal (f) colonies were abundant on agar surfaces when maintained 28 days under lab conditions. (B) In contrast, only bacterial colonies (arrows) were observed at low numbers on soil dilution plates incubated under martian conditions. Compare the microbial growth on labincubated soil dilutions with Colour Lake soils (Fig. 1A; lower left) to the growth of only hypobarophiles (arrows) from the Colour Lake soils incubated at $0.7 \mathrm{kPa}$ (Fig. 1B). (Color graphics available at www.liebertonline.com/ast)

\subsection{Isolation of hypobarophiles from non-soil samples}

After Round 1 assays, hypobarophilic bacterial colonies were observed on agar surfaces used to process human saliva and hand rinsate (volunteer \#1 only) and seawater (coastal surf along Florida on the Merritt Island National Refuge). The hypobarophiles from saliva and hand rinsate were identified as Leuconostoc gasicomitatum, L. gelidum, and L. inhae (Table 2). In contrast, the two colonies observed at low-PTA conditions from seawater were not recovered during Round 2 subculturing assays (Table 1). 
Other samples from coral bean plants, forearm hair, lab benches, and scalp hair were negative for the presence of hypobarophilic bacteria. Fungi were not observed on any of the non-soil samples incubated at low-PTA conditions.

Several of the non-soil sample assays were conducted in a nonquantitative manner, so no estimates of background populations of culturable bacteria or fungi were possible. However, coral bean surfaces $\left(10 \mathrm{~cm}^{2}\right.$ basis $)$ and lab benches $\left(100 \mathrm{~cm}^{2}\right.$ basis) yielded low numbers of bacteria and fungi per unit areas of the assays (Table 1). In contrast, human saliva yielded no culturable fungi and high numbers of culturable bacteria $\left(6.4 \times 10^{6} \mathrm{cfu} / \mathrm{mL}\right)$ from all three volunteers.

\subsection{S rRNA sequencing for confirmed hypobarophiles}

Table 2 lists 76 strains of hypobarophilic bacteria from all sources and is arranged in the following order of source materials: soils, non-soil samples, and type-strains of Serratia with the soils, and then hypobarophiles listed in alphabetical order within each category. Although Table 2 represents primarily confirmed hypobarophiles that successfully completed three rounds of incubation under low-PTA conditions, five strains of the presumptive hypobarophiles Streptomyces aureus and $S$. vinaceus are included for completeness. Assigning taxonomic affiliation at the species level was based on the closest matches being at $\geq 97.5 \% 16 \mathrm{~S}$ sequence identity (Stackebrandt et al., 2002). If the closest matches were either below $97.5 \%$ in the RDP database or the taxonomic affiliations listed in the RDP database lacked species-level identities, the taxonomic affiliations were kept at the genus level.

Hypobarophilic bacteria were identified representing 3 phyla, 10 families, and 11 genera (Table 2), demonstrating the presence of hypobarophiles in diverse and globally distributed ecosystems. The highest numbers of hypobarophiles, with the greatest diversity, were recovered from Colour Lake and Siberian permafrost soils and human saliva (volunteer \#1), representing the genera Carnobacterium, Exiguobacterium, Leuconostoc, Paenibacillus, and Trichococcus. However, many of the hypobarophiles remain to be characterized at the species level. Hypobarophiles are now identified in the families Bacillaceae, Bacillales_Incertae Sedis XII, Carnobacteriaceae, Clostridiaceae, Enterobacteriaceae, Leuconostocaceae, Microbacteriaceae, Norcardiaceae, Paenibacillaceae, and Streptomycetaceae.

\subsection{Growth of type-strains in the genus Serratia under low-PTA conditions}

Six of eight type-strains in the genus Serratia exhibited growth under low-PTA conditions, including $S$. ficaria, $S$. fonticola, S. grimesii, S. liquefaciens, S. plymuthica, and $S$. quinivorans (Fig. 2; Table 3). In contrast, S. marcescens and S. rubidaea failed to grow under all low-temperature and low-pressure conditions; thus the effect of low pressure alone could not be separated from the effect of low temperature. The growth of Carnobacterium inhibens subsp. gilichinskyi, strain WN1359 at low-PTA conditions confirms earlier work on growth of nine type-strains at $0.7 \mathrm{kPa}$ for the genus Carnobacterium, including the observation that WN1359 grew slightly better at the low-PTA conditions at $0.7 \mathrm{kPa}$ when compared to growth under aerobic lab conditions at $101.3 \mathrm{kPa}$ (Nicholson et al., 2013). The growth or lack of growth for the positive and negative controls, respectively (Table 3), matched the growth under low-PTA conditions previously reported for these strains (Nicholson et al., 2013; Schuerger et al., 2013).
FIG. 2. Growth of Serratia spp. on TSA under diverse conditions of pressure, temperature, and gas composition. Cultures were incubated at (A) Earth-lab conditions of $101.3 \mathrm{kPa}, 30^{\circ} \mathrm{C}$, and $\mathrm{O}_{2} / \mathrm{N}_{2}$ atmospheres, (B) low-temperature conditions of $101.3 \mathrm{kPa}, 0^{\circ} \mathrm{C}$, and $\mathrm{O}_{2} / \mathrm{N}_{2}$ atmospheres, (C) low-temperature and high- $\mathrm{CO}_{2}$ conditions of $101.3 \mathrm{kPa}, 0^{\circ} \mathrm{C}$, and $\mathrm{CO}_{2}$-enriched anoxic atmospheres, and (D) Mars conditons of $0.7 \mathrm{kPa}, 0^{\circ} \mathrm{C}$, and $\mathrm{CO}_{2}$-enriched anoxic atmospheres. Control bacterial strains included the following (left to right; bottom row): Bacillus subtilis 168, Carnobacterium inhibens subsp. gilichinskyi WN1359, Escherichia coli ATCC 35218, and Sporosarcina aquamarina SAFN-008. The numbering system goes left to right and from top to bottom (1 through 12) and coincides with the order in Table 3 (from top to bottom). (Color graphics available at www.liebertonline.com/ast)
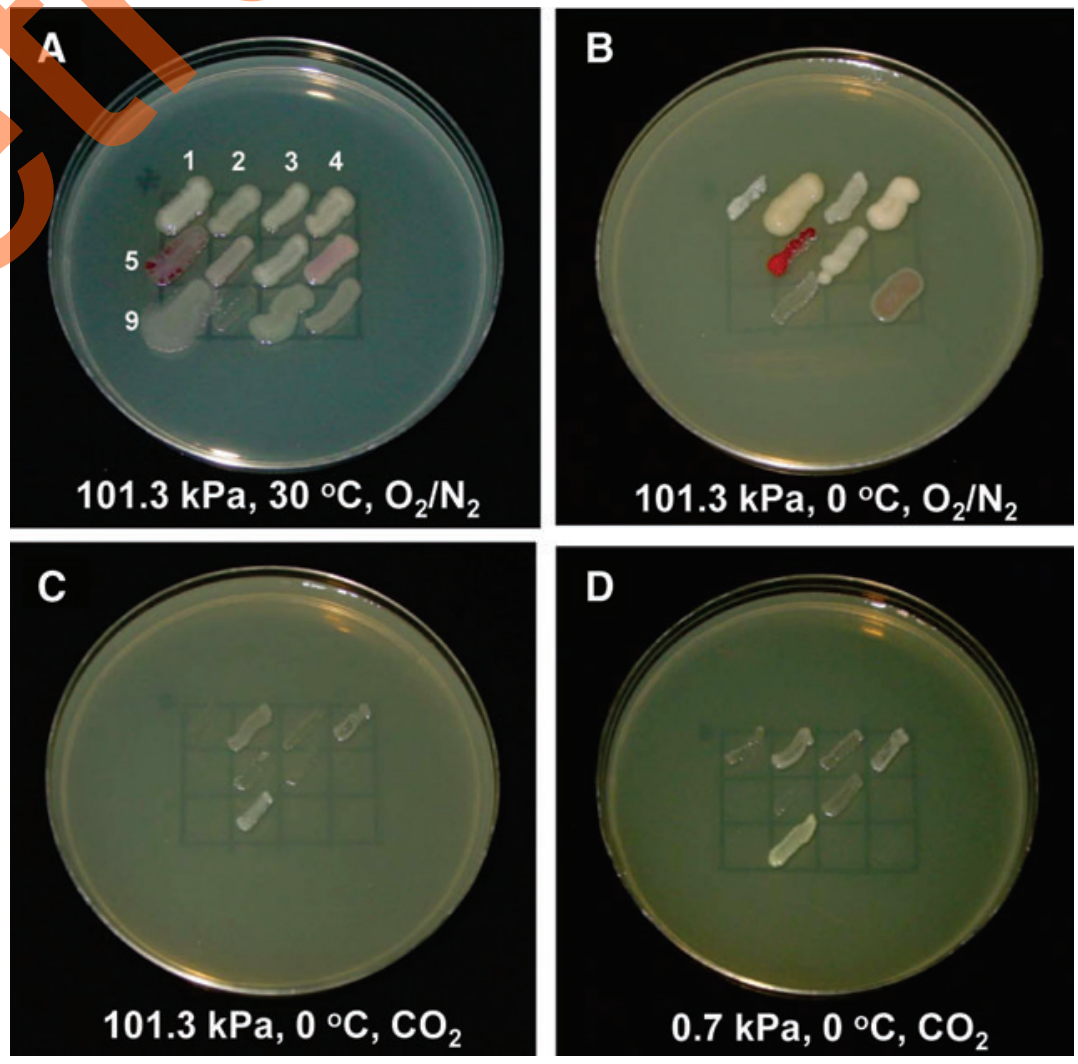
Hypobarophilic bacteria picked from $0.5 \times$ TSA or R2A media maintained under low-PTA conditions were, in general, white and translucent (Fig. 1B). Similarly, six of eight Serratia type-strains that grew under low-PTA conditions lost the pigmentation observed under lab conditions of $101.3 \mathrm{kPa}, 30^{\circ} \mathrm{C}$, and Earth-normal $\mathrm{O}_{2} / \mathrm{N}_{2}$ atmosphere (Fig. 2). In addition, the pigmentation of two strains, Serratia plymuthica and Sporosarcina aquamarina, exhibited increased red pigmentation under a lab pressure of $101.3 \mathrm{kPa}$ when incubated at $0^{\circ} \mathrm{C}$ in a $\mathrm{O}_{2} / \mathrm{N}_{2}$ atmosphere, but then returned to a nonpigmented condition when S. plymuthica was grown at $101.3 \mathrm{kPa}, 0^{\circ} \mathrm{C}$, and $\mathrm{CO}_{2}$-enriched anoxic atmosphere (Fig. 2). Results suggest that both temperature and low $\mathrm{ppO}_{2}$ are responsible for the loss of pigmentation in bacterial cells of Serratia spp. tested under low-PTA conditions. Because maintaining stable hydrated media at $0.7 \mathrm{kPa}$ requires low temperatures at or below $0^{\circ} \mathrm{C}$ (i.e., near the triple-point of water; Haberle et al., 2001), the effects of low pressure alone on cell pigmentation could not be discerned.

\subsection{Growth and colony morphology of permafrost strains at $15^{\circ} \mathrm{C}$ in the presence or absence of oxygen}

Similar to the depigmentation observed at reduced temperatures for the hypobarophiles S. plymuthica and S. aquamarina, multiple strains of Cryobacterium sp. (WN1502 and
WN1485) and Exiguobacterium sibiricum (WN1486, WN1488, WN1492, and WN1493) were observed to lose their yellow/orange pigmentation when grown under anoxic conditions at $15^{\circ} \mathrm{C}$ (Fig. 3). The rest of the permafrost hypobarophile isolates produced rather small $(<1 \mathrm{~mm}$ diameter), cream or light tan colonies and grew either slightly better without oxygen or at approximately the same rate regardless of the presence or absence of oxygen (Fig. 3). Thus, all isolates appeared to be either facultative aerobes or facultative anaerobes. And lastly, all permafrost hypobarophiles picked from soil-dilution plates incubated at low-PTA conditions were not pigmented (similar to Fig. 1B).

\section{Discussion}

The recovery of 23 species in 11 genera of hypobarophilic bacteria from diverse ecosystems supports the conclusion that such low-pressure-adapted bacteria are common on Earth. Most hypobarophiles were detected in arctic, permafrost, and alpine soils and, thus, may indicate that many psychrophiles possess stress regulons that confer dual tolerance to hypobaria. However, hypobarophiles were also recovered from human saliva and hands, and type-strains of the hypobarophilic genera Carnobacterium spp. (current study; Nicholson et al., 2013) and Serratia (current study; Schuerger et al., 2013) were originally recovered from

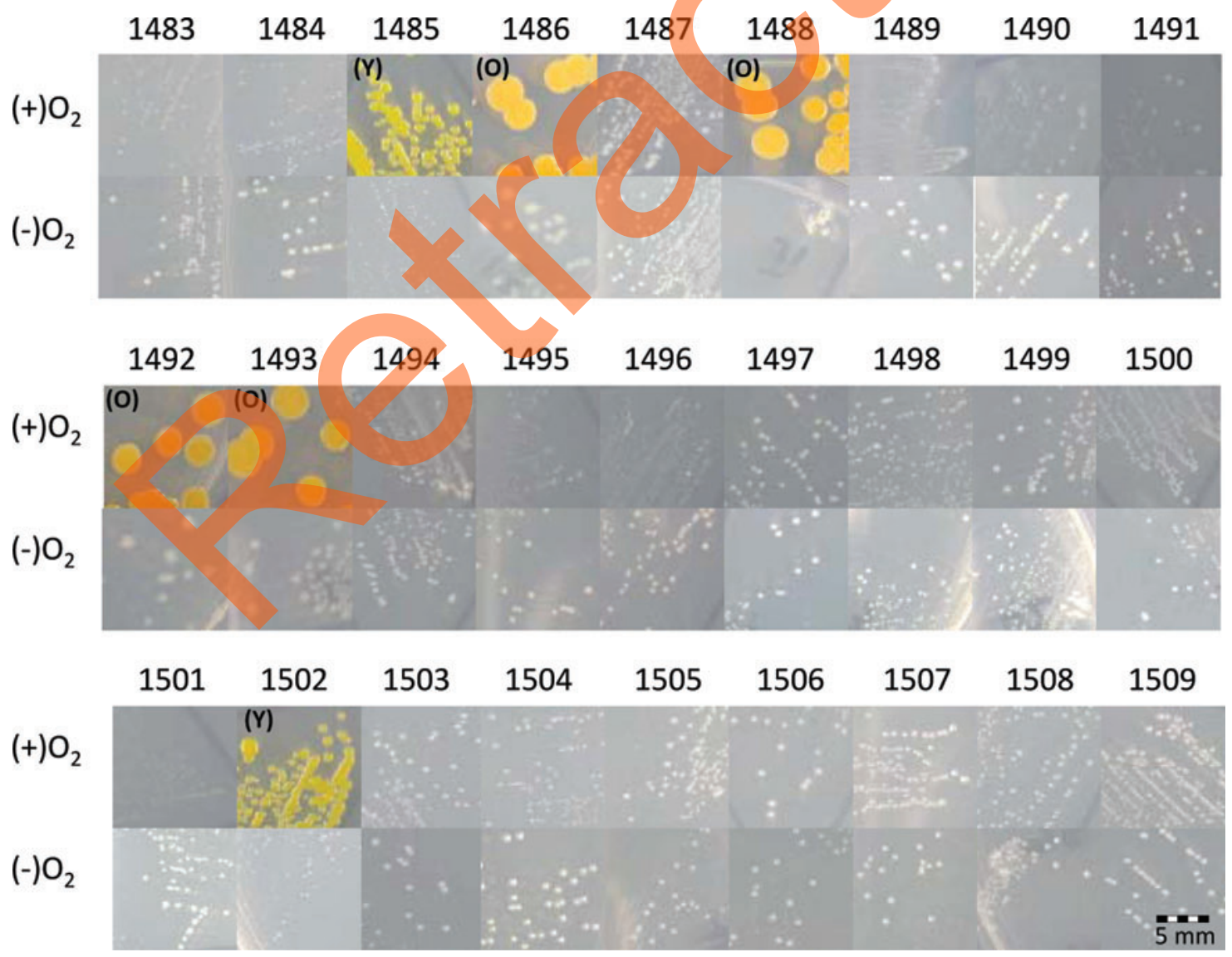

FIG. 3. Growth and colony morphology of permafrost isolates after 7 days of growth at $15^{\circ} \mathrm{C}$. Cultures were incubated on $0.5 \times$ TSA either in the presence $\left[(+) \mathrm{O}_{2}\right]$ or absence $\left[(-) \mathrm{O}_{2}\right]$ of oxygen. Numbers above each paired set of images refer to the WN strain collection numbers in Table 2. Distinctive yellow $(\mathrm{Y})$ and orange $(\mathrm{O})$ pigmented colonies are denoted. Photographs were all taken at the same magnification (see scale bar in lower right corner). (Color graphics available at www.liebertonline.com/ast) 
diverse sources including fig, fish, frozen meat, insect, milk, soil, and water niches (based on cited literature from ATCC and DSMZ culture collections). Thus, hypobarophiles from temperate ecosystems cannot be ruled out. Intriguingly, no archaea (unknown presence) and no fungi (i.e., confirmed to be present in most soil samples under lab, but not low-PTA conditions; Table 1) were recovered in the Round 1 assays, suggesting that the capability to grow at low pressures might be constrained to the domain Bacteria.

All hypobarophilic bacteria except Clostridium sp. exhibited growth under both anoxic (Round 1, 2, and 3 assays) and aerobic (during streak-purification under Earth-normal gases) conditions and, thus, can be labeled as facultative anaerobic hypobarophiles. This interpretation is consistent with other studies that found hypo- and mesobarophilic bacteria capable of growth at pressures between 0.7 and $2.5 \mathrm{kPa}$, respectively, in both anoxic and aerobic low-pressure conditions (Schuerger and Nicholson, 2006; Schuerger et al., 2013). In contrast, two Streptomyces spp. were recovered from arctic soils during three separate iterations of Round 1 assays under low-PTA conditions, but would not grow again in Round 2 assays when streak-purified under lab conditions and retested under lowPTA parameters (Table 2). Also, colonies of presumptive hypobarophiles were observed on several Round 1 assays with alpine soils from Mt. Baker, Washington, and from seawater obtained from coastal surf near the Kennedy Space Center, Florida, but failed to grow during attempts to streak-purify the isolates under lab conditions. Taken together, the failure of subsequent growth at $0.7 \mathrm{kPa}$ for the Streptomyces spp. and presumptive hypobarophiles from Mt. Baker and seawater suggests that geochemical or microbial metabolites from the soils or seawater were required to support growth of these bacteria under low-PTA conditions. To date, no analytical procedures have been reported in the literature that can screen for nonculturable hypobarophiles.

Demonstration that six of eight hypobarophilic typestrains in the genus Serratia could be grown under low-PTA conditions suggests that tolerance to low pressure is a neargenus-wide trait in Serratia. Similar results were reported for the genus Carnobacterium in which nine of nine species type-strains tested were capable of growth under low-PTA conditions (Nicholson et al., 2013). Furthermore, multiple hypobarophilic species were found in the genera Leuconostoc, Paenibacillus, and Trichococcus (Table 2). Taken together, these results suggest that tolerance to low pressure may be widely dispersed in some genera and may not be constrained to unique species.

One of the most intriguing observations in the current work was the recovery of three hypobarophilic Leuconostoc spp. from human saliva and hand rinsate from a single volunteer (three volunteers tested). After the detection of the Leuconostoc spp. from saliva and hands, volunteer \#1 was retested on two separate occasions with negative results. The recovery on only one of three Round 1 assays from a single volunteer suggests that the detection of Leuconostoc hypobarophiles may have been associated with the consumption of food by volunteer \#1 and not indicative of an extant humanassociated hypobarophile microbial community.

Future research should be conducted to expand the list of selective media beyond TSA and R2A for culturing hypobarophiles from environmental samples in order to rule out the role media chemistry might play on recovering hypo- barophilic bacteria from the samples. Although we present evidence that both temperature and gas composition can alter cell pigmentation (Figs. 2 and 3), the loss of cell pigmentation observed for bacterial cells under diverse conditions may have been partly due to the media types used. In previous work (Schuerger and Lee, 2015; Schuerger unpublished), we have used $0.5 \times$ TSA and R2A successfully to recover bacteria and fungi from oligotrophic environments. At this time, we cannot rule out the recovery of hypobarophilic bacteria, archaea, or fungi under low-PTA conditions if other media and incubation protocols are used.

\subsection{Implications for growth of bacteria in Earth's atmosphere}

Numerous papers have reported the recovery of terrestrial bacteria and fungi in Earth's atmosphere, with the accepted maximum altitude of detection at $41 \mathrm{~km}$ (see review by Smith, 2013). But none of the atmospheric sampling studies cited by Smith (2013) examined metabolic activity at the sampled altitudes, or pressure simulations of those altitudes. It remains a goal of aeromicrobiology to probe the upper limits of the active terrestrial biosphere, but such studies have relied upon sampling of probably dormant airborne cells by aircraft or balloons to predict activity. We report here on a series of experiments to recover and identify hypobarophilic bacteria capable of growth at $0.7 \mathrm{kPa}$; the pressure equivalent of $34 \mathrm{~km}$ in the middle stratosphere. Although the experimental conditions were originally designed to simulate the surface of Mars, the results reported here should be applicable to predicting metabolic activity and growth in the terrestrial atmosphere because all bacterial strains, except Clostridium sp., were found to be facultative anaerobes.

Figure 4 depicts the temperature profile of Earth's atmosphere from the surface to $80 \mathrm{~km}$ (based on NASA/ NOAA, 1976). Initially the temperature decreases linearly in the troposphere from the surface to the tropopause (12$15 \mathrm{~km}$ ) with a lapse rate of $6.5^{\circ} \mathrm{C}$ per kilometer. The temperature remains stable near $-60^{\circ} \mathrm{C}$ through the tropopause, increases through the stratosphere approaching $0^{\circ} \mathrm{C}$ at the stratopause, and then decreases to $-80^{\circ} \mathrm{C}$ at the top of the mesosphere. Recent work on temperature minima for metabolic activity and growth in bacteria has demonstrated that some species are capable of cell division and growth down to $-18^{\circ} \mathrm{C}$ and maintenance metabolism down to perhaps $-33^{\circ} \mathrm{C}$ (e.g., Rivkina et al., 2000; Panikov and Sizova, 2007; Clarke et al., 2013; see review by Rummel et al., 2014).

Based on these low-temperature limits for microbial activity and growth, the upper altitude threshold for microbial growth at $-18^{\circ} \mathrm{C}$ is likely between 5 and $6 \mathrm{~km}$ in the troposphere $(54-47 \mathrm{kPa})$ with maintenance metabolism extending perhaps to $7-8 \mathrm{~km}(41-36 \mathrm{kPa})$ (Fig. 4). Recent data (Nicholson et al., 2010; Fajardo-Cavazos et al., 2012; Schuerger et al., 2013; Waters et al., 2014) suggest that pressures down to $5 \mathrm{kPa}$ are not limiting for microbial growth for most species; thus microbial activity will be constrained by other parameters in the atmosphere such as UV irradiation, temperature, desiccation effects, and access to nutrients and liquid water. Based on temperature constraints alone, the only other plausible region in Earth's atmosphere where microbial growth might resume would be 


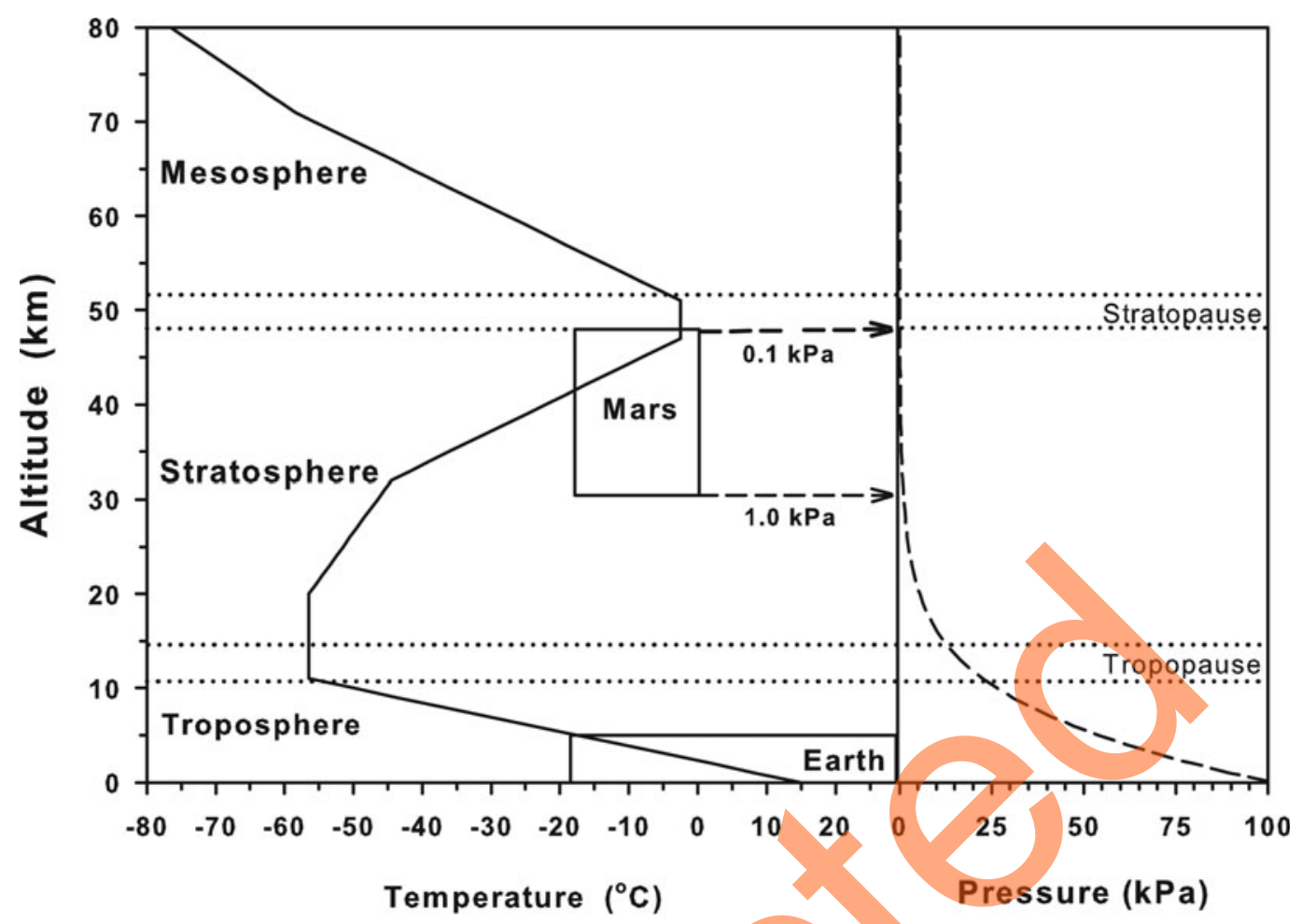

FIG. 4. Temperature (left) and pressure (right) profiles from sea level to $80 \mathrm{~km}$ for a standard Earth atmosphere. The box depicting microbial growth in Earth's troposphere is bounded on the left by a low-temperature threshold of $-18^{\circ} \mathrm{C}$ and extends beyond the graph axis on the right into thermophilic conditions above $100^{\circ} \mathrm{C}$. The box depicting conditions conducive for microbial growth on Mars is bounded on the left by the temperature minimum for growth $\left(-18^{\circ} \mathrm{C}\right)$ and on the right by the stability of liquid water $\left(0^{\circ} \mathrm{C}\right.$ at $\left.0.7 \mathrm{kPa}\right)$. The altitudes in Earth's atmosphere that approximate the surface pressures on Mars are between 30 and $48 \mathrm{~km}(0.1-1.0 \mathrm{kPa})$; thus there is a zone in the upper stratosphere that might fall within temperature and pressure ranges that mimic a portion of the martian surface. (Graph based on the US Standard Atmosphere, 1976; NASA TM-X-74335.)

in the upper stratosphere and stratopause between 40 and $50 \mathrm{~km}$ in which temperature once again approaches $0^{\circ} \mathrm{C}$. However, the pressure range in the upper stratosphere from 40 to $50 \mathrm{~km}$ is $\sim 0.28$ to $0.076 \mathrm{kPa}$ ( 2.8 to $0.76 \mathrm{mbar}$ ), a pressure range that has not yet been shown capable of supporting microbial growth.

Work here with hypobarophiles at $0.7 \mathrm{kPa}$ and other research with mesobarophiles down to $2.5 \mathrm{kPa}$ (Schuerger and Nicholson, 2006; Schuerger et al., 2006, 2013; Nicholson et al., 2010; Fajardo-Cavazos et al., 2012; Waters et al., 2014) suggests that most airborne bacteria are capable of metabolic activity and growth up to the middle troposphere where temperature, not pressure, becomes limiting at 5$8 \mathrm{~km}$. This should hold for bacteria present as individual cells, aggregates of cells, cell/aerosol assemblages, or embedded cells in ice crystals. Although the atmosphere warms up again approaching $0^{\circ} \mathrm{C}$ in the upper stratosphere, it has not yet been demonstrated that bacteria are capable of acquiring nutrients and water under the ultralow pressures in the upper stratosphere near $0.28 \mathrm{kPa}$ to permit cellular metabolism and growth. Thus, the lower to middle troposphere may be designated an airborne microbial habitat as suggested by Diehl (2013), but higher altitudes may serve only as conduits for microbial dispersal between terrestrial and marine ecosystems. To fully characterize the upper limits of the active aerial biosphere on Earth, work must be expanded to probe the absolute minima for microbial metabolism and growth for pressures below $0.7 \mathrm{kPa}$.

\subsection{Implications for growth of bacteria on the surface of Mars}

Robotic missions to potentially habitable destinations on Mars (i.e., called Special Regions; see Rummel et al., 2014) are required to comply with international guidelines of planetary protection as established by the Committee on Space Research (COSPAR) (Bruckner et al., 2009). In addition, guidelines are established to align future human missions to Mars with existing planetary protection protocols for mitigating forward contamination of the surface (Criswell et al., 2005; Race et al., 2008). Critical to planetary protection guidelines for both robotic and crewed missions to Mars is an understanding of how and to what extent terrestrial microorganisms might survive, grow, and evolve on the surface.

Here, we extend to 34 the number of bacterial species capable of growth under martian conditions at $0.7 \mathrm{kPa}$ (current results; Nicholson et al., 2013; Schuerger et al., 2013) and widen the source regions for recovery of hypobarophiles to a diversity of arctic, temperate, and even human ecosystems. However, two key questions must be addressed to properly model the risk of contaminating the 
surface of Mars. First, are hypobarophiles present on spacecraft hardware? And second, if present, will they be able to grow on the actual surface of Mars?

Comparing the species listed in Table 2 with microbial surveys of planetary spacecraft and human missions, several of the hypobarophiles identified here may have been present on space hardware prior to launch. For example, species in the genera Bacillus, Clostridium, Exiguobacterium, Paenibacillus, Rhodococcus, Serratia (including S. liquefaciens), and Streptococcus are reported here as hypobarophiles and have been recovered from robotic spacecraft (La Duc et al., 2003), spacecraft assembly facilities (SAFs) (Moissl et al., 2007; Probst et al., 2010; Vaishampayan et al., 2010), and human missions to the International Space Station or the Mir space station (La Duc et al., 2004; Novikova, 2004). Furthermore, at least three species of Leuconostoc were recovered from human saliva and hand rinsate that exhibited growth at $0.7 \mathrm{kPa}$. Thus, it is reasonable to suggest that hypobarophiles may have been present on some robotic and human spacecraft prior to launch.

Next, can launched hypobarophiles plausibly grow on the surface of Mars? First, our results here and elsewhere (Nicholson et al., 2013; Schuerger et al., 2013) support the conclusion that hypobarophiles are capable of growth under low-PTA conditions near $0.7 \mathrm{kPa}$ that approximate some of the environmental conditions on the surface of Mars. However, the growth rates of the hypobarophiles were extremely slow, and assays often required between 10 and 14 days of growth on continuously stable, hydrated, and nutrient-rich media for small pin-prick-sized colonies to appear on the agar surfaces. Thus, can terrestrial hypobarophiles acquire adequate liquid water and nutrients on the thermodynamically unstable surface of Mars over the course of several sols to carry out metabolism and growth?

Rummel et al. (2014) discussed the thermodynamics of liquid water on the martian surface in order to model the occurrence of Special Regions on Mars, which are defined as "a region within which terrestrial organisms are likely to replicate, or a region which is interpreted to have a high potential for the existence of extant martian life forms." With few exceptions, the martian surface appears to be either too cold $\left(<-18^{\circ} \mathrm{C}\right.$ minimum), too dry $(<0.60$ water activity $\left[a_{\mathrm{w}}\right]$ minimum), or both for growth of terrestrial microorganisms. At low latitudes, surface temperatures can rise above the $-18^{\circ} \mathrm{C}$ minimum threshold for terrestrial microbial growth, but then $a_{\mathrm{w}}$ is significantly below the 0.60 minimum for microbial activity (often $<0.05 a_{\mathrm{w}}$ on Mars). At higher latitudes, $a_{\mathrm{w}}$ may begin to approach conditions conducive for microbial activity ( $>0.60$ ), but the temperature quickly falls below $-18^{\circ} \mathrm{C}$. Several possible exceptions were noted by Rummel et al. (2014), with recurring slope lineae (RSL; most likely caused by subsurface seepage down steep slopes by liquid brines at temperatures $>-20^{\circ} \mathrm{C}$ ), shallow subsurface ices, and partially collapsed lava caves being the most likely to provide stable hydrated niches that might support terrestrial microbial activity and growth. Thus, diurnal and seasonal fluctuations in temperature and $a_{\mathrm{w}}$ are likely to inhibit active metabolism and growth of terrestrial microorganisms on most martian terrains, with possible exceptions of hypobarophiles colonizing locations with stable hydrated brines that fall within temperature and pressure ranges conducive for growth.
One such example of a stable liquid environment near the martian surface may be active RSL recently identified with spectral signatures of hydrated perchlorate and chlorate salts consistent with liquid brines, although other salts could not be ruled out (Ojha et al., 2015). Perchlorates can be both toxic to, and utilized as an energy source by, microorganisms (Coates and Achenbach, 2004). Previously, we have demonstrated the growth of Bacillus subtilis and B. pumilus in hydrated soil solutions extracted from a Mars analog soil modeled after the Phoenix landing site with $1.5 \mathrm{wt} \%$ sodium perchlorate salt (Nicholson et al., 2012). And of the 23 hypobarophilic species described here, we have tested the growth of S. liquefaciens in nonperchlorate salt solutions relevant to the surface of Mars. Vegetative cells of $S$. $l i$ quefaciens were shown capable of growth between $5^{\circ} \mathrm{C}$ and $30^{\circ} \mathrm{C}$ in brines up to $5 \%$ for $\mathrm{MgCl}_{2}$ and $\mathrm{NaCl}$, and up to $10 \%$ for $\mathrm{MgSO}_{4}$ (Berry et al., 2010). The presence of hydrated salts in RSL may provide liquid niches conducive for growth of terrestrial hypobarophiles delivered to the surface by spacecraft, and may provide accessible locations in which to search for extant life on Mars. If hydrated brines are present as shallow subsurface features in active RSL sites, salt tolerance becomes an additional parameter that terrestrial hypobarophiles must overcome in order to grow near the martian surface. Future research should explore interactive effects of low pressures near $0.7 \mathrm{kPa}$, low temperatures required for stable liquid brines at $0.7 \mathrm{kPa}$, and high salt concentrations that mimic surface regolith conditions on Mars.

And lastly, Fig.4 depicts a zone in Earth's upper stratosphere in which temperature is above the microbial growth minimum of $-18^{\circ} \mathrm{C}$ and lies between the pressure range on Mars of $0.1 \mathrm{kPa}$ (top of Olympus Mons) and $1.0 \mathrm{kPa}$ (Hellas Basin). The Mars-like microbial growth zone in Earth's stratosphere is also bounded by $0^{\circ} \mathrm{C}$ at the high end of temperature due to the stability of liquid water at low pressures (Haberle et al., 2001; Rummel et al., 2014). Above $0^{\circ} \mathrm{C}$ at $0.7 \mathrm{kPa}$, liquid water is not stable and sublimates directly from ice to the gaseous phase. Research into microbial activity and growth of hypobarophiles in situ within the terrestrial stratospheric "Mars zone" by longduration experiments on high-altitude balloons or in ground simulations at pressure and temperature conditions present in the stratosphere (current study) will inform planetary protection and astrobiology efforts in locating Special Regions on Mars. We demonstrate here that hypobarophiles are present in diverse terrestrial ecosystems and that there may be zones in both the terrestrial atmosphere and the surface of Mars that may support metabolism and growth of terrestrial microorganisms.

\section{Acknowledgments}

This study was supported in part by grants from the NASA Astrobiology: Exobiology and Evolutionary Biology (NNA08AO15G) and Planetary Protection Research (NNX 12AJ84G) programs to A.C.S. and W.L.N. The authors would like to acknowledge and thank the following individuals for providing soils from diverse ecological niches around the World: K. Krivushin (Siberian permafrost soils), C.P. McKay (Antarctica, Arctic, Atacama Desert, Mojave Desert), D.J. Smith (Mt. Baker, WA), and V. Parro (Río 
Tinto, Spain). The authors do not have any conflicts of interests with vendors, reviewers, or organizations used in conducting the research described herein.

\section{References}

Amato, P., Demeer, F., Melaouhi, A., Fontanella, S., MartinBiesse, A.-S., Sancelme, M., Laj, P., and Delort, A.-M. (2007) A fate for organic acids, formaldehyde and methanol in cloud water: their biotransformation by microorganisms. Atmos Chem Phys 7:4159-4169.

Benardini, J.N., Sawyer, J., Venkateswaran, K., and Nicholson, W.L. (2003) Spore UV and acceleration resistance of endolithic Bacillus pumilus and Bacillus subtilis isolates obtained from Sonoran Desert basalt: implications for lithopanspermia. Astrobiology 3:709-717.

Berry, B.J., Jenkins, D.G., and Schuerger, A.C. (2010) Effects of simulated Mars conditions on the survival and growth of Escherichia coli and Serratia liquefaciens. Appl Environ Microbiol 76:2377-2386.

Bruckner, J., Venkateswaran, K., Osman, S., and Conley, C. (2009) Space microbiology: planetary protection, burden, diversity and significance of spacecraft associated microbes In Encyclopedia of Microbiology, $3^{\text {rd }}$ ed., Elsevier, Oxford, pp 52-65.

Clarke, A., Morris, G.J., Fonseca, F., Murry, B.J., Acton, E., and Price, H.C. (2013) A low temperature limit for life on Earth. PLoS One 8, doi:10.1371/journal.pone.0066207.

Coates, J.D. and Achenbach, L.A. (2004) Microbial perchlorate reduction: rocket-fueled metabolism. Nat Rev Microbiol 2:569-580.

Cole, J.R., Wang, Q., Cardenas, E., Fish, J., Chai, B., Farris, R.J., Kulam-Syed-Mohideen, A.S., McGarrell, D.M., Marsh, T., Garrity, G.M., and Tiedje, J.M. (2009) The Ribosomal Database Project: improved alignments and new tools for rRNA analysis. Nucleic Acids Res 37:D141-D145.

Criswell, M.E., Race, M.S., Rummel, J.D., and Baker, A. (2005) Planetary Protection Issues in the Human Exploration of Mars, NASA/CP-2005-213461, NASA Ames Research Center, Moffett Field, CA.

Diehl, R.H. (2013) The airspace is habitat. Trends Ecol Evol 28:377-379.

Fajardo-Cavazos, P., Waters, S.M., Schuerger, A.C., George, S., Marois, J.J., and Nicholson, W.L. (2012) Evolution of $\mathrm{Ba}$ cillus subtilis to enhanced growth at low pressure: upregulated transcription of des-desKR, encoding the fatty acid desaturase system. Astrobiology 12:258-270.

Haberle, R.M., McKay, C.P., Schaeffer, J., Cabrol, N.A., Grin, E.A., Zent, A.P., and Quinn, R. (2001) On the possibility of liquid water on present-day Mars. J Geophys Res 106:2331723326.

La Duc, M.T., Nicholson, W.L., Kern, R., and Venkateswaran, K. (2003) Microbial characterization of the Mars Odyssey spacecraft and its encapsulation facility. Environ Microbiol 5:977-985.

La Duc, M.T., Sumner, R., Pierson, D.L., Venkat, P., and Venkateswaran, K. (2004) evidence of pathogenic microbes in the International Space Station drinking water: reasons for concern? Habitation 10:39-48.

Lueders, T., Manefield, M., and Friedrich, M.W. (2004) Enhanced sensitivity of DNA- and rRNA-based stable isotope probing by fractionation and quantitative analysis of isopycnic centrifugation gradients. Environ Microbiol 6: 73-78.
Michiels, C., Bartlett, D.H., and Aertsen, A. (2008) HighPressure Microbiology, ASM Press, Washington, DC.

Moissl, C., Osman, S., La Duc, M.T., Dekas, A., Brodie, E.L., DeSantis, T.Z., and Venkateswaran, K. (2007) Molecular bacterial community analysis of clean rooms where spacecraft are assembled. FEMS Microbiol Ecol doi:10.1111/ j.1574-69.2007.00360.x.

NASA/NOAA. (1976) US Standard Atmosphere, 1976, NASA TM-X-74335/NOAA-S/T-76-1562, U.S. Government Printing Office, Washington, DC.

Nicholson, W.L., Fajardo-Cavazos, P., Fedenko, J., Ortiz-Lugo, J.L., Rivas-Castillo, A., Waters, S.M., and Schuerger, A.C. (2010) Exploring the low-pressure growth limit: evolution of Bacillus subtilis in the laboratory to enhanced growth at 5 kilopascals. Appl Environ Microbiol 76:7559-7565.

Nicholson, W.L., McCoy, L.E., Kerney, K.R., Ming, D.W., Golden, D.C., and Schuerger, A.C. (2012) Aqueous extracts of a Mars analogue regolith that mimics the Phoenix landing site do not inhibit spore germination or growth of model spacecraft contaminants Bacillus subtilis 168 and Bacillus pumilus SAFR-032. Icarus 220:904-910.

Nicholson, W.L., Krivushin, K., Gilichinsky, D., and Schuerger, A.C. (2013) Growth of Carnobacterium spp. from permafrost under low pressure, temperature, and anoxic atmosphere has implications for Earth microbes on Mars. Proc Natl Acad Sci USA 110:666-671.

Nicholson, W.L., Zhalnina, K., Oliveira, R.R., and Triplett, E.W. (2015) Proposal to rename Carnobacterium inhibens to Carnobacterium inhibens subsp. inhibens subsp. nov., and description of Carnobacterium inhibens subsp. gilichinskyi subsp. nov., a novel psychrotolerant bacterium isolated from Siberian permafrost. Int J Syst Evol Microbiol 65:556-561.

Novikova, N.D. (2004) Review of the knowledge of microbial contamination of the Russian manned spacecraft. Microb Ecol 47:127-132.

Ojha, L., Wilhelm, M.B., Murchie, S.L., McEwen, A.S., Wray, J.J., Hanley, J., Masse, M., and Chojnacki, M. (2015) Spectral evidence for hydrated salts in recurring slope lineae on Mars. Nat Geosci 8:829-832.

Panikov, N.S. and Sizova, M.V. (2007) Growth kinetics of microorganisms isolated from Alaskan soil and permafrost in solid media frozen down to -35 degrees C. FEMS Microbiol Ecol 59:500-512.

Probst, A., Vaishampayan, P., Osman, S., Moissl-Eichinger, C., Andersen, G.L., and Venkateswaran, K. (2010) Diversity of anaerobic microbes in spacecraft assembly clean rooms. Appl Environ Microbiol 76:2837-2845.

Race, M.S., Kminek, G., and Rummel, J.D. (2008) Planetary protection and humans on Mars: NASA/ESA workshop results. Adv Space Res 42:1128-1138.

Rivkina, E.M., Friedmann, E.I., McKay, C.P., and Gilichinsky, D.A. (2000) Metabolic activity of permafrost bacteria below the freezing point. Appl Environ Microbiol 66:3230-3233.

Rummel, J.D., Beaty, D.W., Jones, M.A., Bakermans, C., Barlow, N.G., Boston, P.J., Chevrier, V.F., Clark, B.C., de Vera, J.-P., Gough, R.V., Hallsworth, J.E., Head, J.W., Hipkin, V.J., Kieft, T.L., McEwen, A.S., Mellon, M.T., Mikucki, J.A., Nicholson, W.L., Omelon, C.R., Peterson, R., Roden, E.E., Lollar, B.S., Tanaka, K.L., Viola, D., and Wray, J.J. (2014) A new analysis of Mars "Special Regions": findings of the Second MEPAG Special Regions Science Analysis Group (SR-SAG2). Astrobiology 14:887-968. 
Santl-Temkiv, T., Finster, K., Hansen, B.M., Pasic, L., and Karlson, U.G. (2013) Viable methanotrophic bacteria enriched from air and rain can oxidize methane at cloud-like conditions. Aerobiologia 29:373-384.

Schuerger, A.C., and Lee, P. (2015) Microbial ecology of a crewed rover traverse in the high Arctic: Low microbial dispersal and implications for planetary protection on human Mars missions. Astrobiology 15:478-491.

Schuerger, A.C. and Nicholson, W.L. (2006) Interactive effects of hypobaria, low temperature, and $\mathrm{CO}_{2}$ atmospheres inhibit the growth of mesophilic Bacillus spp. under simulated martian conditions. Icarus 185:143-152.

Schuerger, A.C., Berry, B.J., and Nicholson, W.L. (2006) Terrestrial bacteria typically recovered from Mars spacecraft do not appear able to grow under simulated martian conditions [abstract 1397]. In 37 th Lunar and Planetary Science Conference, Lunar and Planetary Institute, Houston.

Schuerger, A.C., Ulrich, R., Berry, B.J., and Nicholson, W.L. (2013) Growth of Serratia liquefaciens under $7 \mathrm{mbar}, 0^{\circ} \mathrm{C}$, and $\mathrm{CO}_{2}$-enriched anoxic atmospheres. Astrobiology 13:115131.

Sharma, A., Scott, J.H., Cody, G.D., Fogel, M.L., Hazen, R.M., Hemley, R.J., and Huntress, W.T. (2002) Microbial activity at gigapascal pressures. Science 295:1514-1516.

Smith, D.J. (2013) Microbes in the upper atmosphere and unique opportunities for astrobiology research. Astrobiology 13:981-990.

Stackebrandt, E., Fredrickson, J.K., Garrity, G.M., Grimont, P.A.D., Kämpfer, P., Maiden, C.J., Nesme, X., Roselló-Mora, R., Swings, J., Trüper, H.G., Vauterin, L., Ward, A.C., and Whitman, W.B. (2002) Report of the ad hoc committee for the re-evaluation of the species definition in bacteriology. Int J Syst Evol Microbiol 52:1043-1047.

Vaishampayan, P., Osman, S., Andersen, G., and Venkateswaran, K. (2010) High-density $16 \mathrm{~S}$ microarray and clone librarybased microbial community composition of the Phoenix spacecraft assembly clean room. Astrobiology 10:499-508.
Vaitilingom, M., Deguillaume, L., Vinatier, V., Sancelme, M., Amato, P., Chaumerliac, N., and Delort, A.-M. (2013) Potential impact of microbial activity on the oxidant capacity and organic carbon budget in clouds. Proc Natl Acad Sci USA 110:559-564.

Waters, S.M., Robles-Martinez, J.A., and Nicholson, W.L. (2014) Exposure of Bacillus subtilis to low pressure (5 kilopascals) induces several global regulons, including those involved in the SigB-mediated general stress response. Appl Environ Microbiol 80:4788-4794.

Address correspondence to: Andrew C. Schuerger University of Florida Space Life Sciences Laboratory 505 Odyssey Way

Merritt Island, FL 32953

E-mail: schuerg@ufl.edu

Submitted 4 August 2015 Accepted 25 November 2015

Abbreviations Used
$\mathrm{cfu}=$ colony-forming units
low-pressure $(0.7 \mathrm{kPa})$, low-temperature
$\left(0^{\circ} \mathrm{C}\right)$, and $\mathrm{CO}_{2}$-enriched anoxic
$\quad\left(\right.$ low $\left.\mathrm{ppO}_{2}\right)$

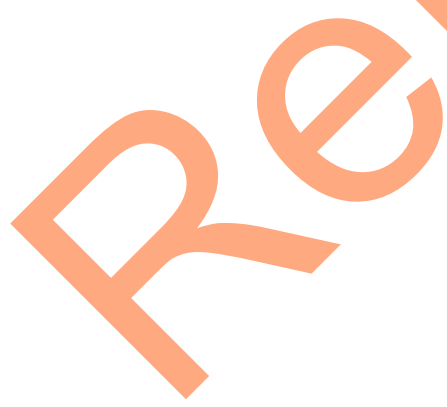

